\title{
Assessing the effectiveness of dialkylcarbamoylchloride (DACC)-coated post-operative dressings versus standard care in the prevention of surgical site infection in clean or clean-contaminated, vascular surgery (the DRESSINg trial): study protocol for a pilot feasibility randomised controlled trial
}

Joshua P. Totty ${ }^{*}$ (D, Amy E. Harwood, Paris L. Cai, Louise H. Hitchman, George E. Smith and lan C. Chetter

\begin{abstract}
Background: Surgical site infection in vascular surgery has a reported incidence of up to $19 \%$. A novel method of reducing this rate of infection is dressings coated with dialkylcarbamoylchloride (DACC), a hydrophobic wound contact layer that binds bacteria and removes them from the wound bed. Early research has suggested that DACC-coated wound dressings are effective in reducing surgical site infection when applied to wounds healing by primary intention post-operatively, therefore this trial aims to assess the feasibility of producing high-quality evidence assessing this theory.
\end{abstract}

Methods: Patients undergoing clean or clean-contaminated vascular surgery will be randomised to have their surgical wounds dressed with a DACC-coated dressing or a non-coated occlusive absorbent post-operative dressing. All other aspects of their peri-operative care will be standardised or carried out in line with hospital policy. Wound assessments will be carried out between day 5-7, day 30 ( \pm 3 days) and 6 months post-operatively ( \pm 7 days) by a blinded assessor using the ASEPSIS scoring tool. Quality of life data using EQ-5D and SF-36, resource use and mortality data will also be collected. This feasibility trial will dictate the conduct of a full-scale trial through the collection of data on recruitment and retention rates, and fitness-for-purpose of the follow-up arrangements.

Discussion: Surgical site infections are now the second most common hospital acquired infections with a significant cost implication. The aim of the DRESSINg trial is to investigate the effectiveness of a novel preventative measure at reducing wound infections post-surgery and will provide robust evidence to support or deny its use.

Trial registration: Clinicaltrials.gov identifier: NCT02992951, Registered 12/12/16. REC Reference: 16/LO/2135.

Keywords: Infection, Surgery, Surgical wound, Dialkylcarbamoylchloride, DACC, Prevention

* Correspondence: hy9jpt@hyms.ac.uk

Academic Vascular Surgical Unit, Hull Royal Infirmary, Anlaby Road, Hull HU3

2JZ, UK 


\section{Introduction}

The Centres for Disease Control and Prevention (CDC) defines a surgical site infection (SSI) as an infection within 30 days of an operation or up to 90 days if an implant is left in place and the infection is related to an operative procedure [1]. SSI's occur in at least 5\% [2] of patients and have a significant impact on patient morbidity, mortality and have subsequent time and cost implications [3].

Microorganisms causing SSI's may be either endogenous (present on the patient) or exogenous (from the environment). The most common bacteria causing a superficial wound infection are those which are present on the skin of the patient. Deeper wound infections may be due to contamination of the wound by bacteria encountered during a procedure. Despite the reported incidence of SSI's for clean surgery of $2.1 \%$ [4], the incidence in practice varies significantly. Open varicose vein surgery has an incidence of SSI's reported in the literature which varies from 1.5 to $16 \%[5,6]$ whilst figures from the surgical site infection surveillance demonstrated a high rate of SSI's in patients undergoing lower limb amputation (13.1\%) [7]. Conversely, SSI rates following carotid endarterectomy are reported as low as $0.2 \%[8,9]$.

The Sorbact ${ }^{\circ}$ range of dressings contains dialkylcarbamoylchloride (DACC), found in a spider's web, which mediates the irreversible binding of bacteria that exhibit a high cell surface hydrophobicity (CSH). Large numbers of adherent or 'trapped' bacteria can then be removed from the wound at each dressing change. They are removed without disrupting the bacterial cell wall, thereby avoiding the resultant increase in inflammation observed with traditional antibiotics or antiseptics [10]. Numerous bacteria and fungi that exhibit CSH have been shown to attach to the DACC-coated material, including Staphylococcus aureus, Pseudomonas aeruginosa, Enterococcus faecalis, Candida albicans and the dermatophyte Trichophyton rubrum $[11,12]$.

DACC-coated dressings are available on the open market in both the UK via the National Health Service (NHS) supply chain, and the USA [13].

The current available evidence, although limited, favours the use of DACC-coated dressings in reducing SSI [14]. A recently published randomised controlled trial and preceding pilot study concluded that in a cohort of women undergoing caesarean section in a single centre, DACC-coated dressings proved to be clinically and cost-effective in reducing the rates of SSI $[15,16]$, indicating that further high-quality studies are needed in this promising area to assess outcomes in a more diverse patient population.

\section{Methods/design}

\section{Study objectives}

The aim of this pilot randomised trial is to evaluate the feasibility of conducting a fully powered randomised controlled trial to investigate the effectiveness of DACC-coated post-operative dressings in the reduction of surgical site infection in vascular surgical patients, using a fraction (one-fifth) of the patients required for a fully powered RCT.

\section{Study design and setting}

This is a pilot feasibility randomised single-centre clinical trial in the setting of a University Teaching Hospital, based in the UK, offering tertiary vascular surgery services. The protocol has been prepared in line with the Standard Protocol Items: Recommendations for Interventional Trials (SPIRIT) guidelines [17].

\section{Inclusion criteria}

The inclusion criteria are:

1. Over 18 years of age

2. Undergoing clean or clean-contaminated vascular surgery (as opposed to contaminated or dirty surgery, in which there is infection already present).

3. Able to understand (i.e. have sufficient language skills) and complete the patient information sheet, consent forms, and questionnaires.

4. Able and willing to give informed consent to participate in the trial (patients may be capacitous but unwilling to give informed consent; they would not meet the inclusion criteria).

\section{Exclusion criteria}

The exclusion criteria are:

1. Patients actively taking antibiotics for other conditions up to the day of surgery (not including surgical prophylaxis or antibiotic use related to the index procedure)

2. Patients undergoing carotid endarterectomy (as these patients have an infection rate significantly lower than rates seen in other types of vascular surgeries $[8,9]$ )

3. Allergies to any component of either the DACCcoated dressing or the control dressing

4. Inability to give informed consent due to incapacity (as defined by the MCA 2005 [18])

5. Aged under 18 years at the time of recruitment

6. Use of investigational drug/device therapy within preceding 4 weeks that may interfere with this study.

DACC-coated post-operative dressings and post-operative procedures

Patients will receive standardised care pre-operatively. Hair removal (clipping) and anaesthesia will be 
conducted according to local hospital policy; in our centre, hair is clipped, not shaven, immediately prior to the procedure in the operating theatre. Skin preparation will be standardised to Povidone-iodine in aqueous solution, unless there is a documented patient allergy. Prophylactic antibiotics will be given as per local guidelines and recorded in the patient notes. Patients will be randomised on the day of their surgical procedure to receive post-operative dressing with a DACC-coated dressing (Leukomed ${ }^{\circ}$ Sorbact $^{\circ}$, BSN Medical, Hull, UK), or a non-coated occlusive absorbent control post-operative dressing (standard practice) (OpSite ${ }^{\circ}$ Post-Op, Smith \& Nephew, Hull, UK).

All dressings will be replaced on day 2 post-procedure, and again at the time of first wound review, which takes place between post-operative days 5-7. Interim dressing changes will be permitted where there is a clinical indication such as soiling or damage to the dressing. On discharge from hospital, patients will be provided with further dressings of the same variety to ensure like-for-like dressing changes up to the point of wound healing, as well as instructions to community teams that the only wound dressings to be used are the ones provided for trial purposes, in order to maintain treatment adherence.

\section{Outcomes}

Outcomes will be divided into two sections: feasibility outcomes and clinical outcomes.

\section{Feasibility outcomes}

- The measured effect size of the trial intervention, in order to contribute to the power calculation and design of a full scale RCT

- Eligibility rates and reasons for non-eligibility

- Participant recruitment rates and reasons for nonrecruitment

- Follow-up and study retention rates and reasons for drop-out/non-attendance

- Fitness for purpose and acceptability of follow-up arrangements

- The suitability of the trial intervention in different wound types/areas

- The suitability of the inclusion/exclusion criteria

- The suitability of outcome assessment measure(s)

- Fitness for purpose and acceptability of data collection methods, including the use of smartphone or online 'apps.'

- Rates of participant withdrawal from the trial; participant response rates to questionnaires; likely rates of missing study data.

\section{Clinical outcomes-primary outcome}

The primary outcome will be the incidence of SSI-measured by total ASEPSIS score $\geq 21$ [19] — within 30 days of surgery, or as defined by the CDC definition of SSI $[1,20]$.

\section{Secondary outcomes}

Secondary outcomes will be:

- The incidence of SSI-total ASEPSIS score $\geq 21$ or SSI as defined by CDC definitions-at 90 days for implant patients only (as per the CDC definition for SSI in implant-involving surgery)

- Satisfactory healing-total ASEPSIS score $\leq 10$-at 30 days post-surgery for non-implant surgery and implant patients

- Satisfactory healing-total ASEPSIS score $\leq 10$-at 90 days for implant patients only

- Quality of life: using EurolQol 5 Domains (EQ-5D-3 L) and Short Form 36 (SF-36) v2 questionnaires at 5-7 days, $30 \pm 3$ days, 3 months \pm 7 days, and 6 months \pm 7 days.

- Time to return to normal activity/work (measured in working days between the day of surgery and the day the patient returned to work).

- Resource use-primary care visits, requirement for antibiotics, readmission, re-intervention within 30 days and 6 months.

- 30-day mortality.

\section{The ASEPSIS scale}

The ASEPSIS scale is a standardised tool used to determine the presence of SSI (with a score $\geq 21$ ) or impaired wound healing (score $>10$ but $<21$ ) [19]. The ASEPSIS tool has been reported to be repeatable and related to outcome $[21,22]$. A score of $<10$ is indicative of satisfactory wound healing.

\section{Quality of life assessments}

The EQ-5D questionnaire is a widely recognised and validated generic measure of health-related quality of life. This questionnaire has been assessed for acceptability and validity in a number of patient groups [23, 24]. The SF-36 has been well-validated in a variety of UK populations $[25,26]$. It measures eight domains which can be used to calculate summary physical and mental component scores.

\section{Trial timescales}

It is anticipated that the recruitment and treatment phase will be completed within 18 months. The primary endpoint is measured after 30 days, with all patients followed up for 6 months after entry into the trial. 


\section{Sample size calculation}

The incidence of SSI's following clean and clean contaminated, non-implant surgery varies widely between the procedures to be included in this study. Whilst the overall infection rate is quoted as being $2.1-3.3 \%$, this is predominantly based on the findings of Cruse and Foord [27] following their long-term surveillance. This has been criticised as it predominantly relies on questionnaire follow-up, over the phone at day 28 post-surgery. The reality in practice is that the infections rates are much higher and are more frequently diagnosed when examined by a blinded observer [28].

The power calculation is based on the primary endpoint having a $90 \%$ power and $5 \%$ significance. A non-randomised prospective trial comparing Leukomed ${ }^{\circ}$ Sorbact ${ }^{\oplus}$ with standard dressings in non-implant, clean or clean-contaminated vascular surgery patients, carried out in the same centre demonstrated an overall reduction in SSI from 19 to $10 \%$ [29]. To demonstrate the same reduction in SSI, a sample size of 320 per group, or a total of 712 patients, allowing for a $10 \%$ loss to follow-up, is required. Study retention rates will be examined at the first interim analysis, and the power calculation amended accordingly. With such a large sample size, there is a high potential for larger loss to follow-up rates than initially expected. Therefore, this pilot study will aim to recruit one-fifth (144) of the total patients needed. The results of this pilot trial, along with other published data on DACC in the reduction of SSI, will be combined to produce a final power calculation for a full-scale RCT.

\section{Recruitment}

Patients that are referred for a clean or clean-contaminated vascular surgical operation will be considered for the study. Patients may be identified at their initial outpatient appointment, at pre-assessment clinics or on their admission to the ward. Patients admitted on a semi-elective or emergency basis will be identified as they are admitted to the ward. Suitable patients will be made aware of the study and provided with the appropriate information, including the patient information sheet. Following this, patients that express an interest will be invited to a screening visit with a study investigator. For inpatients, this visit may take place on the inpatient ward. At this appointment, patients will be assessed against the inclusion and exclusion criteria, and if potential participants meet said criteria, informed consent to participate in the trial will be taken by a study doctor and recorded on the informed consent form and in the patient notes.

To ensure confidentiality and to adhere to data protection guidelines, participants will be given a unique identifying number which does not allow for the identification of study arms or demographic information. The identifiable patient information will not be available to any person(s) outside of the research group. The patient's general practitioner will also be informed of study enrolment and study details. No changes to concomitant medications will be made, and no restrictions to trial treatments or methods based on concomitant treatment will be made.

\section{Randomisation}

Consented patients will be randomised to one of the two parallel testing groups (DACC-coated dressing or standard post-operative dressing) by equal randomisation using the online computerised sealed enveloped method (Sealed Envelope, London, UK) (see Fig. 1).

\section{Blinding}

Due to the nature of the treatment, a double-blind study is not possible (Leukomed ${ }^{\circ}$ Sorbact $^{\bullet}$ contains a coloured wound contact layer that is not present on standard dressings). To reduce the risk of bias, assessor reported outcomes, namely the ASEPSIS scoring of wounds, will be performed by a blinded assessor who will not have access to the patient notes. Anonymous photos of wounds will be rated by a third blinded assessor to reduce bias and ensure discrepancies in ASEPSIS scores are addressed. Bias in other outcomes will be limited by the use of predetermined objective measurements and bias in quality of life outcomes will be reduced by using patient-reported quality of life outcomes.

\section{Data collection}

All patient data, including patient reported quality of life and clinical outcomes, will be entered into a paper-based case report form (CRF) and anonymised into Microsoft Excel for further analysis and monitored by the research and development department. Only the principal and co-investigators will have access to the full dataset.

\section{Study visits}

Following screening and informed consent, a baseline visit will take place to record demographic data and medical history, prior to randomisation. Study visits will take place between post-op days 5 and 7, at post-op day 30 and at 6 months. Patients will be contacted both by post and telephone when arranging follow-up in order to maximise retention. At the study visits, information such as antibiotic use, length of hospital stay, visits to the general practitioner, dressing changes/number of dressings used and return to work or daily activities will be recorded by a non-blinded study nurse or doctor. Patients will be deemed to have returned to normal activities if they have returned to work (if they are of working age) or if they have returned to their pre-operative activity levels. In particular, patients will be asked if they have returned to driving, exercise or previous levels of social activity. 


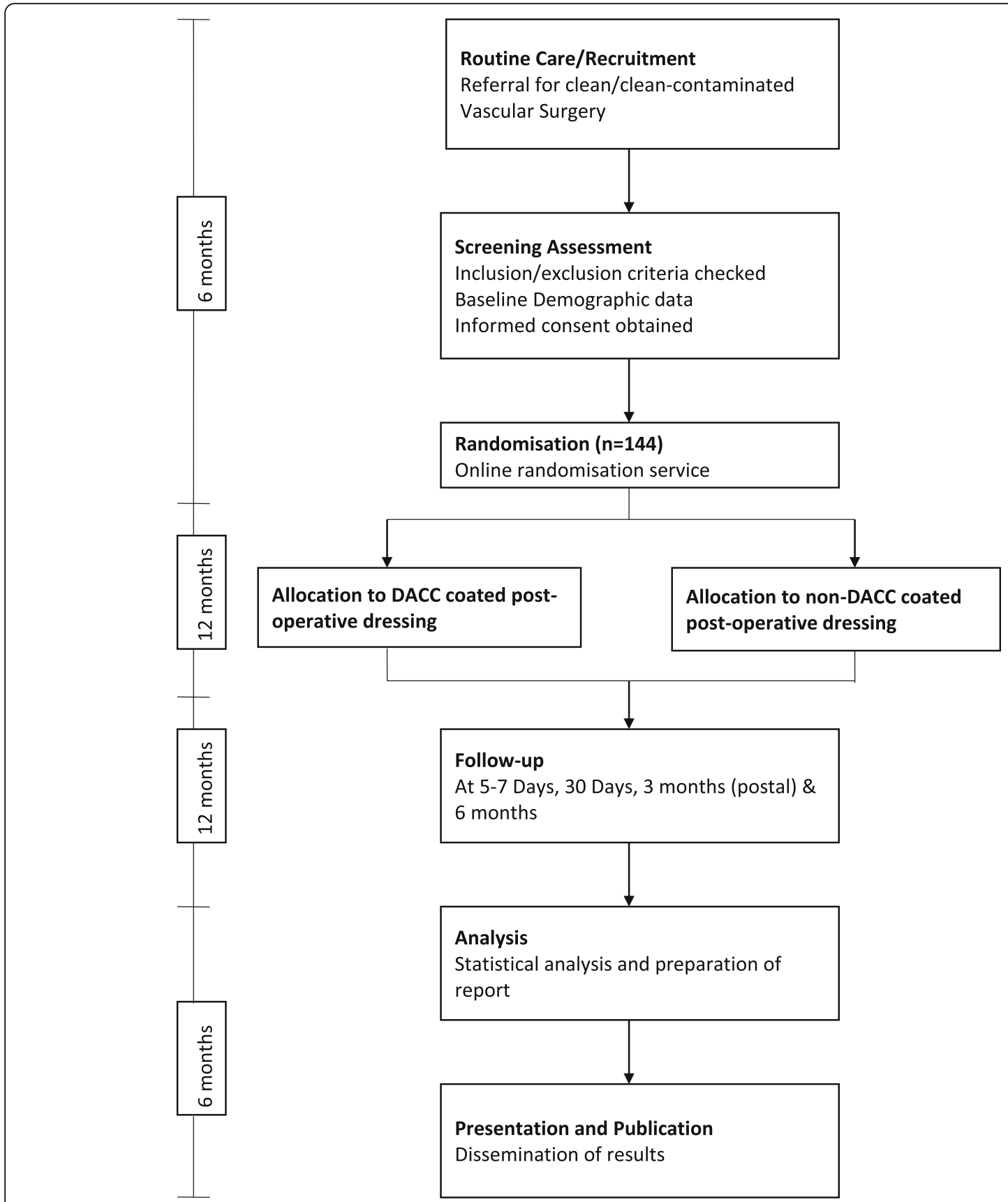

Fig. 1 Study flow chart. The target sample size for the pilot phase of this study is 144 . Treatment and follow-up will run concurrently, giving a total time of 24 months from study start to completion

The same individual will remove the dressing in situ and dispose of it in an opaque bag. A second assessor, blinded to the dressing type, will be invited into the room in order to score the wound on the ASEPSIS scale. This will be recorded in the CRF. With the dressing removed, anonymous photographs will be taken of the wound, to be reviewed by an assessor not involved in trial recruitment or follow-up and used for the standardisation of ASEPSIS scores between participants. With the blinded assessor out of the room, the first study nurse or doctor will replace the dressing. Postal quality of life questionnaires will be sent to participants at 3 months and 6 months. Table 1 outlines the schedule of assessments.

\section{Trial exit}

Patients will exit the trial after completing the 1-year follow-up visit, if they experience an adverse event or reaction deemed to require their withdrawal from the trial 
Table 1 Schedule of assessments (SPIRIT figure)

\begin{tabular}{|c|c|c|c|c|c|c|c|}
\hline \multirow[t]{2}{*}{ Visit } & 1 & 2 & 3 & 4 & 5 & - & - \\
\hline & Screening & $\begin{array}{l}\text { Baseline } \\
\text { assessment }\end{array}$ & $\begin{array}{l}\text { Day 0- day of } \\
\text { surgery }\end{array}$ & $\begin{array}{l}\text { Day } 5-7 \\
\text { post op }\end{array}$ & $\begin{array}{l}\text { Day } 30 \text { post } \\
\text { op } \pm 3 \text { days }\end{array}$ & $\begin{array}{l}3 \text { Months post- } \\
\text { op } \pm 7 \text { days }\end{array}$ & $\begin{array}{l}6 \text { Months post- } \\
\text { op } \pm 7 \text { days }\end{array}$ \\
\hline Screening questionnaire & $x$ & & & & & & \\
\hline Patient information sheet & $x$ & & & & & & \\
\hline Informed consent & & $x$ & & & & & \\
\hline Baseline questionnaire & & $x$ & & & & & \\
\hline SF-36 & & $x$ & & $x$ & $x$ & $x$ & $x$ \\
\hline EQ-5D-3 L & & $x$ & & $x$ & $x$ & $x$ & $x$ \\
\hline GP letter & & $x$ & & & & & \\
\hline RANDOMISATION FORM & & & $x$ & & & & \\
\hline $\begin{array}{l}\text { HPA surgical wound healing post } \\
\text { discharge questionnaire }\end{array}$ & & & & & $x$ & & $x$ \\
\hline Clinical review of wound & & & & $x$ & $x$ & & $x$ \\
\hline
\end{tabular}

SF36 short form 36 utility, EQ-5D EuroQoL 5 domain utility index, HPA health protection agency

or if they choose to withdraw at any point during their inclusion in the trial.

\section{Statistical analysis}

Data will be analysed on an intention to treat basis. The SPSS (IBM Corporation, Armonk, NY, USA) computer package will be used with a two-sided $p$ value of $<0.05$ taken as the level of significance. For feasibility outcomes, simple categorical data will be presented descriptively using mean (SD), median (IQR) for skewed data or $n$ (\%) for each group. Recruitment of a large trial within 18 months (based on rates observed in the pilot trial), and retention of $85 \%$ or better will be deemed acceptable.

Descriptive statistics (mean (sd) and $n(\%)$ ) will be calculated for demographic and baseline characteristics of the patients. For the clinical outcome of SSI at 30 days, logistic regression analysis will be undertaken with SSI as the dependent variable and randomisation group as the independent variable. The model will be adjusted for confounding variables such as sex, age, BMI, smoking status, diabetes status and surgical site, as well as implant vs non-implant surgery. The regression model performance will be assessed by the Hosmer and Lemeshow test. Logistic regression will also be undertaken for satisfactory healing-total ASEPSIS score $\leq 10$. For quality of life (EQ-5D-3 L and SF-36), an intragroup and intergroup analysis will be performed, using Friedman's two-way analysis of variance (ANOVA) test to assess for intragroup differences, and Mann-Whitney $U$ tests to assess for intergroup differences of the SF-36 responses. For the EQ-5D, responses will be dichotomised into 'no problems' and 'problems', and intragroup analysis conducted using related sample's Cochrane's Q test, with Pearson's $X^{2}$ tests for intergroup analysis. For time to event data (time to return to work and mortality), Kaplan-Meier and long rank tests will be used to calculate and compare event rates between groups, followed by a cox regression to adjust for confounding variables.

\section{Monitoring, safety and quality control}

The study will be monitored in accordance with the local research and development department's standard operating procedures to ensure compliance with The International Council for Harmonisation of Technical Requirements for Pharmaceuticals for Human Use (ICH) Good Clinical Practice (GCP) [30] and the Research Governance Framework 2005.

The collection and reporting of data on adverse events and serious adverse events will be in accordance with $\mathrm{ICH}$ GCP and the Research Governance Framework 2005. An adverse event (AE) is any untoward medical occurrence in a subject to whom a medicinal product or device has been administered as part of a research study, including occurrences which are not necessarily caused by or related to a medicinal product or device. An adverse reaction (AR) is any untoward and unintended response in a subject to a medicinal product or device. An adverse event becomes serious (SAE) if it results in death, is life-threatening, requires hospitalisation or prolongation of existing hospitalisation, results in persistent or significant disability or incapacity, is a congenital anomaly or birth defect or is otherwise considered medically significant by the investigator. The term 'life-threatening' refers to an event in which the patient was at risk of death at the time of the event; it does not refer to an event which hypothetically might have caused death if it were more severe.

Hospitalizations planned prior to enrolment in the trial (elective surgery) or for social reasons should not normally be considered as SAEs unless the hospitalisation has to be prolonged.

The AE reporting period for this trial begins as soon as patients have consented to the trial and ends at the point 
of discharge from the trial, following a final study visit or telephone consultation at 1 year following surgery. The health status of subjects will be checked at each study visit. The investigator will record all directly observed AEs and all AEs spontaneously reported by the trial subject. A pre-existing condition (i.e. a disorder present at the baseline study visit and noted on the baseline medical history/ physical examination form/medical notes) is not to be reported as an AE unless the condition worsens or episodes increase in frequency during the AE-reporting period. All adverse events (serious and non-serious) will be recorded by the investigator in patient's data collection forms (CRFs) using R\&D's adverse event report form. All adverse events will be recorded by the investigator in patients' medical records/notes. All AEs will be followed-up by investigators until the event has resolved or a decision has been taken for no further follow-up.

If a clinically significant abnormal laboratory value occurs, this abnormality will be recorded as an adverse event/reaction.

The investigator will report fatal or life-threatening SAEs or serious adverse reactions (SARs) to the research ethics committee (REC) within 7 days and follow-up information within a further 8 days by following the request on the serious event initial and follow-up report forms. The investigator will send all other SAE or SAR reports to the REC within a maximum of 15 days.

Patients have access to information on the complaints procedure for obtaining compensation following harm through non-negligence or negligence as a result of participating in the trial.

Study completion refers to the date of final data collection from the last patient. Paper records from the trial will be stored for 5 years from trial end.

An end of study declaration form will be submitted to the REC and Trust R\&D within 90 days from completion of the trial and within 15 days if the trial is discontinued prematurely. A summary of the trial report/ publication will be submitted to the REC and Trust $R \& D$ within 1 year of the end of trial.

\section{Discussion}

Surgical site infections are now the second most common hospital acquired infections, and cost many thousands of pounds per patient per infection [31]. Any strategy for reducing this burden should be investigated. DACC-coated dressings show no evidence of antimicrobial resistance and have no documented cases of adverse reaction. The aim of the DRESSINg trial is to investigate the effectiveness of this novel preventative measure at reducing wound infections post-surgery.

\section{Trial status}

The DRESSINg trial pilot phase began recruiting in January 2017 and is currently in active recruitment.

\section{Abbreviations}

AE: Adverse event; AR: Adverse reaction; CRF: Case report form; CSH: Cell surface hydrophobicity; DACC: Dialkylcarbamoylchloride; EQ-5D: EuroQol 5 domains; GP: General practitioner; HPA: Health protection agency; HYMS: Hull York Medical School; ICF: Informed consent form; MCA: Mental Capacity Act; R\&D: Research and development; REC: Research ethics committee;

SAE: Serious adverse event; SAR: Serious adverse reaction; SSI: Surgical site infection; UK: United Kingdom

\section{Acknowledgements}

Not applicable.

\section{Trial sponsor}

Hull and East Yorkshire Hospitals NHS Trust, R\&D Department, Office 13, 2nd Floor Daisy Building, Castle Hill Hospital, Castle Rd., Cottingham, East Yorkshire HU16 5JQ. The sponsor has no role in study design, data collection or interpretation or dissemination of findings.

\section{Funding}

Funding is provided by the Academic Vascular Surgery Department, Hull York Medical School.

\section{Availability of data and materials}

Not applicable.

\section{Authors' contributions}

JPT is a Clinical Research Fellow in Vascular Surgery and Co-Investigator, responsible for study development, ethical approval, patient recruitment, data collection, statistical analysis, manuscript preparation and manuscript writing. AEH is a Doctoral Research Fellow, responsible for manuscript preparation and revision, study development and ethical approval. PLC is a Clinical Research Fellow in Vascular Surgery, responsible for patient recruitment, data collection, manuscript preparation and manuscript writing. $\mathrm{LHH}$ is an Academic Foundation Doctor in Vascular Surgery, responsible for patient recruitment, data collection, manuscript preparation and manuscript writing. GES is an NIHR Academic Clinical Lecturer in Vascular Surgery and Chief Investigator, responsible for study development, ethical approval, manuscript writing and manuscript revision. ICC is a consultant in Vascular Surgery and Professor of Surgery at Hull York Medical School, responsible for study development and supervision, ethical approval and manuscript revision. All authors read and approved the final manuscript. No professional writers were used in the preparation of this manuscript, nor will be used in the preparation of manuscripts for publication of the final study data.

\section{Ethics approval and consent to participate}

Ethical approval was granted by London-Harrow Research Ethics Committee on 23/11/2016. REC reference 16/LO/2135 with the IRAS project ID 215973. The Research Protocol, patient information sheet and informed consent form have been approved by the Hull and East Yorkshire Hospitals NHS Trust Research and Development department (R2034). Informed consent via an information sheet and signed consent form is gained before enrolment into the trial, and the presence of a valid, signed and dated informed consent form is required for participation in the trial. All enrolled participants will have access, should they request it, to the full study protocol, and any publication of the final results.

\section{Consent for publication}

The participant information sheet, which has been approved by the Research Ethics Committee, provides information that the results will be submitted for publication in a scientific journal and a final report written. Participants will not be identified in any of the reports or publications. Coauthorship will be assigned according to the *Recommendations for the Conduct, Reporting, Editing, and Publication of Scholarly Work in Medical Journals, Updated December 2015 of the International Committee of Medical Journal Editors.

Competing interests

The authors declare that they have no competing interests. 


\section{Publisher's Note}

Springer Nature remains neutral with regard to jurisdictional claims in published maps and institutional affiliations.

Received: 20 June 2018 Accepted: 10 January 2019

Published online: 18 January 2019

\section{References}

1. Surgical site infection (SSI) event. https://www.cdc.gov/nhsn/pdfs/ pscmanual/9pscssicurrent.pdf. Accessed 16 Jan 2019

2. National Institute for Health and Care Excellence. Surgical Site Infection (NICE Guideline 74). 2008. Available at https://www.nice.org.uk/guidance/ cg74/resources/surgical-site-infections-prevention-and-treatment-pdf975628422853. Accessed 16 Jan 2019

3. Owens CD, Stoessel K. Surgical site infections: epidemiology, microbiology and prevention. J Hosp Infect. 2008;70 Suppl 2:3-10.

4. Culver DH, Horan TC, Gaynes RP, Martone WJ, Jarvis WR, Emori TG, Banerjee $S N$, Edwards JR, Tolson JS, Henderson TS. Surgical wound infection rates by wound class, operative procedure, and patient risk index. National Nosocomial Infections Surveillance System. Am J Med. 1991:91:152S-7S.

5. Hirsemann S, Sohr D, Gastmeier K, Gastmeier P. Risk factors for surgical site infections in a free-standing outpatient setting. Am J Infect Control. 2005;33:6-10.

6. Hayden A, Holdsworth J. Complications following re-exploration of the groin for recurrent varicose veins. Ann R Coll Surg Engl. 2001;83:272-3.

7. Elgohari S, Thelwall S, Lamagni T, Sheridan E, Charlett A. Surveillance of surgical site infections in NHS hospitals in England. Public Health England. 2014;29

8. Greenstein AJ, Chassin MR, Wang J, Rockman CB, Riles TS, Tuhrim S, Halm EA. Association between minor and major surgical complications after carotid endarterectomy: results of the New York carotid artery surgery study. J Vasc Surg. 2007:46:1138-46.

9. Stone PA, Srivastava M, Campbell JE, Mousa AY, Hass SH, Kazmi H, Dearing DD, AbuRahma AF. A 10-year experience of infection following carotid endarterectomy with patch angioplasty. J Vasc Surg. 2011;53:1473-7.

10. Mosti G, Magliaro A, Mattaliano V, Vascular C, Picerni P, Angelotti N. Comparative study of two antimicrobial dressings in infected leg ulcers: a pilot study. J Wound Care. 2015:24:4-9.

11. Bullough L, Little G, Hodson J, Morris A. The use of DACC-coated dressings for the treatment of infected, complex abdominal wounds. Wounds UK 2012:8:102-9.

12. Cooper R, Jenkins L. Binding of two bacterial biofilms to dialkyl carbamoyl chloride (DACC)-coated dressings in vitro. J Wound Care. 2016;25(2):76, 7882. https://doi.org/10.12968/jowc.2016.25.2.76.

13. Leukomed Sorbact. https://www.bsnmedical.co.uk/products/wound-carevascular/category-product-search/advanced-wound-care/infectionmanagement/leukomedr-sorbactr.html. Accessed 16 Jan 2019.

14. Totty JP, Bua N, Smith GE, Harwood AE, Carradice D, Wallace T, Chetter I. Dialkylcarbamoyl chloride (DACC)-coated dressings in the management and prevention of wound infection: a systematic review. J Wound Care. 2017:26:107-14

15. Stanirowski PJ, Bizoń M, Cendrowski K, Sawicki W. Randomized controlled trial evaluating dialkylcarbamoyl chloride impregnated dressings for the prevention of surgical site infections in adult women undergoing cesarean section. Surg Infect. 2016;17:427-35

16. Stanirowski PJ, Kociszewska A, Cendrowski K, Sawicki W. Dialkylcarbamoyl chloride-impregnated dressing for the prevention of surgical site infection in women undergoing cesarean section : a pilot study. Arch Med Sci. 2016;12(5):1036-42.

17. Chan A, Tetzlaff JM, Altman DG, et al. Spirit 2013 statement: defining standard protocol items for clinical trials. Ann Intern Med. 2013;158:200-7.

18. Department of Health. Mental capacity act. London: HMSO; 2005.

19. Wilson APR, Sturridge MF, Treasure T, Grüneberg RN. A scoring method (asepsis) for postoperative wound infections for use in clinical trials of antibiotic prophylaxis. Lancet. 1986:327:311-2.

20. Horan TC, Gaynes RP, Martone WJ, Jarvis WR, Emori TG. CDC definitions of nosocomial surgical site infections, 1992: a modification of CDC definitions of surgical wound infections. Infect Control Hosp Epidemiol. 1992;13:606-8.

21. Armitage P, Berry G, Matthews JN. Statistical methods in medical research. Wiley; 2002. p. 817

22. Byrne DJ, Malek MM, Davey PG, Cuschieri A. Postoperative wound scoring. Biomed Pharmacother. 1989;43:669-73.
23. Johnson JA, Pickard AS. Comparison of the EQ-5D and SF-12 health surveys in a general population survey in Alberta, Canada. Med Care. 2000;38:115-21.

24. HME v A, Essink-Bot M-L, PFM K, Bonsel GJ. Test-retest reliability of health state valuations collected with the EuroQol questionnaire. Soc Sci Med. 1994;39:1537-44.

25. Jenkinson C, Wright L, Coulter A. The SF 36 health survey questionnaire. ...if used within its limits. BMJ. 1993;307:449.

26. Jenkinson C, Stewart-Brown S, Petersen S, Paice C. Assessment of the SF-36 version 2 in the United Kingdom. J Epidemiol Community Health. 1999;53: $46-50$.

27. Cruse PJ, Foord R. The epidemiology of wound infection. A 10-year prospective study of 62,939 wounds. Surg Clin North Am. 1980;60:27-40.

28. Leaper D. Use of antibiotic prophylaxis in clean non-implant wounds. J Antimicrob Chemother. 1998:41:501-4.

29. Bua N, Smith GE, Totty JP, Pan D, Wallace T, Carradice D, Chetter IC. Dialkylcarbamoyl chloride dressings in the prevention of surgical site infections after nonimplant vascular surgery. Ann Vasc Surg. 2017;44:387-92.

30. ICH Guidelines. http://www.ich.org/products/guidelines.html. Accessed 16 Jan 2019.

31. Under the Knife Report: taking a zero tolerance approach to preventable surgical site infections in UK hospitals. Available at http://www.carefusion.co. uk/documents/international/continuing-education/infection-prevention/IP_ Under-the-Knife_CE_EN.pdf. Accessed 16 Jan 2019.
Ready to submit your research? Choose BMC and benefit from:

- fast, convenient online submission

- thorough peer review by experienced researchers in your field

- rapid publication on acceptance

- support for research data, including large and complex data types

- gold Open Access which fosters wider collaboration and increased citations

- maximum visibility for your research: over $100 \mathrm{M}$ website views per year

At BMC, research is always in progress.

Learn more biomedcentral.com/submissions 